\title{
A possibility of remote detection of air breakdown in a focal spot of microwave beam using reflected signal
}

\author{
V.E. Semenov ${ }^{1}$, E.I. Rakova ${ }^{1}$, M.Yu. Glyavin ${ }^{1}$, G.S. Nusinovich ${ }^{2}$ \\ ${ }^{1}$ Institute of Applied Physics, Russian Academy of Sciences, Nizhny Novgorod, Russia, \\ ${ }^{2}$ IREAP, University of Maryland, College Park, Maryland 20742-3511, USA
}

\section{Introduction}

Propagation of high power microwave beam through atmosphere can initiate the breakdown which results in formation of plasma reflecting the incident microwave radiation. The breakdown delay time, as well as the plasma parameters, is sensitive to existence of radioactive materials which can ionize air producing thereby seed electrons in the vicinity of the breakdown prone volume [1-3]. Therefore a study of the breakdown evolution can give some particular information about the closeness of radioactive materials to the focal spot of high power microwave beam [3, 4]. The necessary information can be obtained using a reflection of the incident microwave radiation from the breakdown plasma.

In this paper the breakdown process was simulated numerically using the same model as in [2]. Calculations of the reflection evolution of the incident microwave beam were completed for the breakdown in atmospheric air. In this case the frequency of electron collisions with molecules as well as the electron diffusion coefficient and frequency of electron attachment to oxygen molecules. A dependence of frequency of the electron impact ionization on amplitude of electric field is also fixed and results of breakdown simulation are determined mainly by intensity of the incident microwave beam, microwave frequency, $f$, length, $l_{f}$, of the microwave beam focal region, initial distribution of plasma density in space, and intensity of the external source of ionization which is produced by cosmic rays and radioactive material in the vicinity of the focal region.

\section{Results}

Simulations were completed assuming that the amplitude of the microwave electric field in the focal point is twice the breakdown threshold. Two different frequencies $f_{1}=110 \mathrm{GHz}$ and $f_{2}=670 \mathrm{GHz}$ where considered in simulations which corresponds to the frequencies of gyrotrons used in MIT experiments [5] and in IAP-UMD experiments [6], respectively. Two different scenarios of breakdown were studied. In the first scenario, the breakdown started from well localized initial plasma that can be interpreted as a result of stochastic ionization by single particle of cosmic ray. The external ionization source was set to zero in this case. Within the second scenario, we started with the zero initial plasma density, while an action of a permanent weak source of external ionization was taken into account. In the simulations, the main attention was paid to evolution of the reflection coefficient of incident microwave beam from the breakdown plasma.

The simulation results are presented below in Figs. 1-4.
As follows from these figures, the reflection evolution depends on parameters and can be rather complicated. However the common peculiarity of this evolution is associated with a well pronounced peak of the reflection coefficient at the initial stage of breakdown. The duration of this peak is determined mainly by a value of the impact ionization frequency in the undisturbed field of microwave beam at its focal point. In case of the first breakdown scenario (localized peak of initial plasma density), the maximum value of the reflection coefficient is not very sensitive to the microwave frequency or focal length, $l_{f}$ (see Fig. 1 and 2). The reflection coefficient reaches the same peak value $G \approx 0.35$ almost independently on frequency, focal length, and maximum value of initial plasma density.
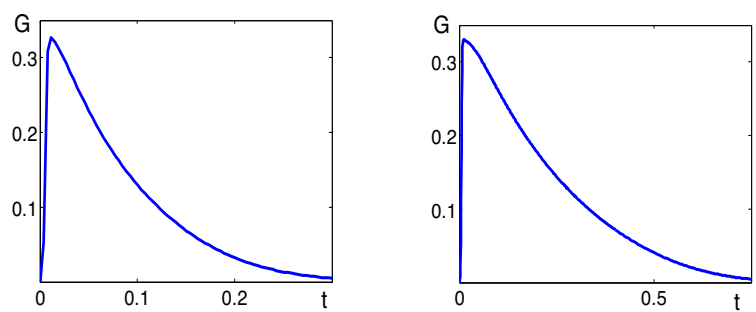

Fig. 1. The amplitude reflection coefficient, G, vs. the time $t$ (in $\mu$ s). Simulations were performed for the atmospheric air and microwave frequency $f_{2}=670 \mathrm{GHz}$. The first breakdown scenario was considered (zero source of external ionization) The left and right panels present simulation results for $l_{f} \approx 2.3 \mathrm{~mm}$ and $l_{f}$ $\approx 7 \mathrm{~mm}$, respectively

In the case of the second breakdown scenario, the maximum value of reflection coefficient is sensitive mainly to a value of the parameter

$$
\eta=\frac{N_{s} \delta}{N_{c}\left(1+\delta^{2}\right)} \cdot \exp (1 / g),
$$

where $N_{s}$ stands for plasma density created by external ionization source in atmospheric air under equilibrium conditions (without any microwave radiation), $\delta=v / \omega$ is the electron collision frequency normalized to circular microwave frequency, $g=k l_{f}$ is the focal length normalized to vacuum wave number. When this parameter is small $(\eta<<1)$, an intensity of the external ionization source does not affect the maximum value of the reflection coefficient (see Fig. 3). However, transition to the opposite limiting case $(\eta>>1)$ results in considerable decrease in the reflection coefficient (sf. Fig. 1 and 4). The further increase in the intensity of the external ionization source is accompanied only by slow logarithmic decrease in the reflection coefficient. 
It should be noted that in the long time evolution of the reflection coefficient there is a qualitative difference between the cases of higher and lower microwave frequency (cf. Fig. 1 and 2). In previous studies [2, 3] it was shown that at a higher frequency the discharge evolution is associated with continuous propagation of the ionization front, whereas at a lower frequency this evolution is associated with quasi-periodic jumps of the ionization front. The continuous propagation of the ionization front is accompanied by smooth evolution of the reflection coefficient (Fig. 1), whereas the jumps of the ionization from are accompanied by oscillations in this coefficient (Fig. 2).
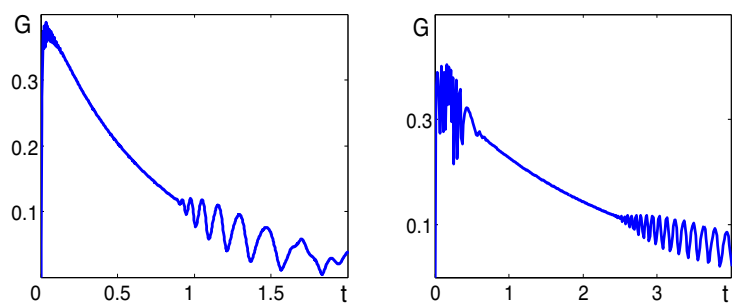

Fig. 2. The amplitude reflection coefficient, G, vs. the time the time $t$ (in $\mu \mathrm{s}$ ). Simulations were performed for the atmospheric air and microwave frequency $f_{l}=110 \mathrm{GHz}$. The first breakdown scenario was considered. The left and right panels present simulation results for $l_{f} \approx 1.4 \mathrm{~cm}$ and $l_{f} \approx 4.3 \mathrm{~cm}$, respectively
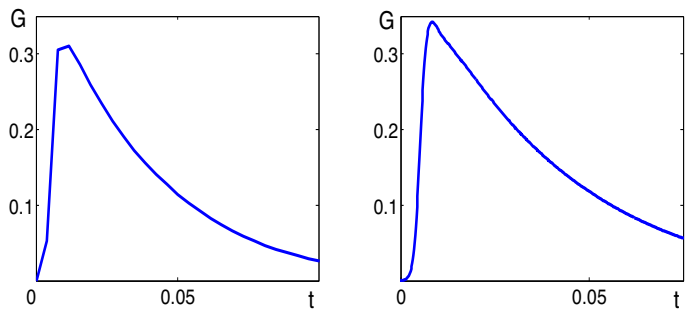

Fig. 3. The dependence of the amplitude reflection coefficient $\mathrm{G}$ on the time $t$ (in $\mu \mathrm{s}$ ). Simulations were performed for $f=$ $670 \mathrm{GHz}$ and $l_{f} \approx 0.7 \mathrm{~mm}$. The left panel presents simulation results in the case of zero external source of ionization and the right panel presents simulations results in the case of zero initial plasma density and intensity of the external ionization source which corresponds to the parameter $\eta \approx 0.01$
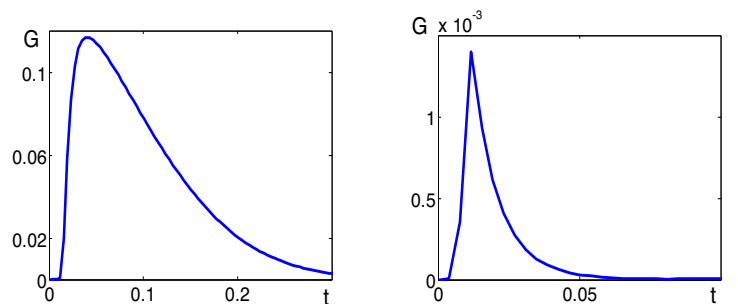

Fig. 4. The amplitude reflection coefficient, G, vs. the time $t$ (in $\mu \mathrm{s}$ ). The microwave frequency $f=670 \mathrm{GHz}$. Both left and right panels present simulation results in case of the second breakdown scenario with the same intensity of the external ionization source. However the focal lengths are different and the corresponding values of the parameter $\eta$ are very different: $l_{f} \approx$ $2.3 \mathrm{~mm}$. $\mathrm{x}$ (the parameter $\eta \approx 2 \cdot 10^{7}$ ) on the left panel whereas $l_{f} \approx 7 \mathrm{~mm}$ (the parameter $\eta \approx 10^{37}$ ) on the right panel

\section{Acknowledgements}

The work was supported by the RFBR, project No. 17-02-00183.

\section{References}

1. V.E. Semenov, E.I. Rakova, V.P. Tarakanov, M.Yu. Glyavin, G.S. Nusinovich // Physics of Plasmas, 2015, 22, 092308

2. V.E. Semenov, E.I. Rakova, M.Yu. Glyavin, and G. S. Nusinovich // Physics of Plasmas, 2016, 23, 073109

3. G.S. Nusinovich, P. Sprangle, V.E. Semenov, D.S. Dorozhkina, M.Yu. Glyavin // J. Appl. Phys., 2012, 111, 124912

4. D. Kim, D. Yu, A. Sawant, M.S. Choe, I. Lee, S.G. Kim, E.M. Choi. Remote detection of radioactive material using highpower pulsed electromagnetic radiation // Nature Communications, 2017, 8, 15394

5. A. Cook, M. Shapiro, R. Temkin. Pressure dependence of plasma structure in microwave gas breakdown at $110 \mathrm{GHz}$ // Applied Physics Letters 2010, 97, 1, 011504

6. M.Yu. Glyavin, A.G. Luchinin, A.A. Bogdashov, V.N. Manuilov, M.V. Morozkin, Yu. Rodin, G.G. Denisov, D. Kashin, G. Rogers, C.A. Romero-Talamas, R. Pu, A.G. Shkvarunetz, G.S. Nusinovich. Experimental study of the pulsed terahertz gyrotron with record-breaking power and efficiency parameters // Radiophysics and Quantum Electronics 56, 2014, 8-9, 497-507 